\title{
Neutrophil-to-lymphocyte ratio and mean platelet volume in the diagnosis of bacterial infections in COVID-19 patients. A preliminary analysis from Ecuador
}

\author{
Jorge Luis Vélez-Páez'1,2, Wendy Tercero-Martínez¹, Glenda Jiménez-Alulima1, \\ Johanna Navarrete-Domínguez ${ }^{1}$, Luis Cornejo-Loor ${ }^{1}$, Christian Castro-Bustamante ${ }^{1}$, \\ Miguel Cabanillas-Lazo ${ }^{3,5}$, Joshuan J. Barboza ${ }^{4,5}$, Alfonso J. Rodriguez-Morales 6 ,7,8,9 \\ ${ }^{1}$ Unidad de Cuidados Intensivos, Centro de Investigación Clínica, Hospital Pablo Arturo Suárez, Quito, Ecuador; \\ ${ }^{2}$ Facultad de Ciencias Médicas, Universidad Central del Ecuador, Quito, Ecuador; \\ ${ }^{3}$ Facultad de Medicina, Universidad Nacional Mayor de San Marcos, Lima, Peru; \\ ${ }^{4}$ Escuela de Medicina, Universidad Señor de Sipán, Chiclayo, Peru: \\ ${ }^{5}$ Tau-Relaped Group, Trujillo, Peru; \\ ${ }^{6}$ Crupo de Investigación Biomedicina, Faculty of Medicine, Fundación Universitaria Autónoma de Las Americas, \\ Pereira, Risaralda, Colombia; \\ IInstitución Universitaria Visión de las Américas, Pereira, Risaralda, Colombia; \\ ${ }^{8}$ School of Medicine, Universidad Privada Franz Tamayo, Cochabamba, Bolivia; \\ ${ }^{9}$ Faculty of Health Sciences, Universidad Cientifica del Sur, Lima, Peru
}

Article received 19 July, 2021; accepted 23 November, 2021

\section{SUMMARY}

Introduction: Hospitalized COVID-19 patients are at risk of hospital infection. The neutrophil-to-lymphocyte ratio (NLR), lymphocyte-C-reactive protein ratio (LCR) and mean platelet volume (MPV) are established inflammation markers reflecting the systemic inflammatory response. The objective of this study was to evaluate the clinical characteristics of patients with COVID-19 and bacterial co-infections, as well as the correlation with NLR and MPV.

Methods: We assessed the role of the NLR and MPV in diagnosing bacterial infections in COVID-19 patients. The Wilcoxon test was used to compare the mean NLR and MPV between the diagnostic evaluation moments, while the Mann-Whitney test was used to compare NLR and MPV by sex and age.

Results: The NLR was compared three days before the culture and the day of taking the culture, observing significant differences $(\mathrm{p}=0.020)$. MPV three days before the culture and the day of the culture were compared, also observing significant differences $(\mathrm{p}=0.031)$. NLR and MPV were compared at the different evaluation times according to sex and age group, observing for the age group significant differences for the NLR three days before the culture $(\mathrm{p}=0.004)$.

Conclusion: In our study, there were significant differences in NLR and MPV between the three days before culture and the day of culture. It is advisable to continue to enrol more patients in the study so that in the future, we can add results on the diagnostic accuracy of the NLR and MPV in the timely diagnosis of bacterial infection in patients with COVID-19.

Keywords: Neutrophil-to-lymphocyte ratio; mean platelet volume; COVID-19; Bacterial infections; coinfection.

Corresponding author

Alfonso J. Rodriguez-Morales

E-mail: arodriguezmo@cientifica.edu.pe 


\section{INTRODUCTION}

$\mathrm{H}$ ospitalized COVID-19 patients are at permanent risk of hospital infection [1-3]. In addition, accumulating evidence suggests that severe COVID-19 patients may have dysregulation of the immune response that allows viral hyperinflammation [4]. Therefore, all patients with severe COVID-19 should be screened for hyperinflammation using laboratory parameters [5]. The neutrophil-to-lymphocyte ratio (NLR), lymphocyteC-reactive protein ratio (LCR) and mean platelet volume (MPV) are established markers of inflammation that reflect the systemic inflammatory response [6].

Blood tests play an essential role in the early diagnosis of the disease, especially for those with an inflammatory process. In the research carried out by Usul et al., through a diagnostic precision study, the role of biomarkers in peripheral blood samples in diagnosing COVID-19 in emergency services was evaluated [7]. Considering a limit value $<1.8$, the NLR had a sensitivity of $59.5 \%$ and a specificity of $76.5 \%$ (AUC 0.739 ; 95\% CI $0.684-$ $0.79 ; \mathrm{p}<0.001)$, so the authors concluded that the NLR is helpful for the diagnosis of COVID-19, since it is an inflammatory marker, although with low sensitivity and specificity [7]. Another study, conducted by Zhang et al., performed a model to predict the risk of developing severe pneumonia in patients with early-stage COVID-19. Investigators demonstrated that the NLR in patients with severe pneumonia was significantly higher than in patients with non-severe pneumonia (Median 7.43, IQR 3.35-19.07 vs Median 2.9, IQR 1.64-4.08; $\mathrm{p}<0.001)$ [8].

The MPV, which is the measurement of platelet size, and the neutrophil-to-lymphocyte ratio (NLR), which is easily calculated by dividing the absolute neutrophil count by the absolute lymphocyte count, are reported in the routine blood count and have been shown to have an excellent value for indicating the general inflammatory status of a patient [9]. Patients diagnosed with COVID-19, especially in severe cases, have a higher NLR and a higher white blood cell count with fewer lymphocytes [10]. Although it has become clear that the MPV and the NLR play a predictive role in infection, mortality, and inflammation in patients with COVID-19, it has not been possible to establish an exact limit value.
The objective of this study was to evaluate the clinical characteristics of patients with COVID-19 and bacterial co-infections, as well as the correlation with NLR and MPV.

\section{METHODS}

\section{Study design}

An observational, analytical, retrospective study of diagnostic assessment was carried out from the intensive care unit (ICU) database of the Pablo Arturo Suárez Hospital, Quito, Ecuador.

\section{Eligibility criteria}

A cohort of hospitalized patients, with 72 hours at the intensive care unit, with the diagnosis of COVID-19 by RT-PCR, or positive pulmonary tomography (CO-RADS 4-5) was developed. Inclusion criteria included: need of mechanical ventilation and presenting with suspected bacterial infection (by culture, positive bacterial isolation collected 72 hs before) associated with an increase in the SOFA score in 24 hours by 2 points, an acute change in the characteristics of tracheal secretions to abundant purulent secretions, new-onset shock with distributive characteristics and hypoxemia in the absence of pulmonary embolism documented by angiography pulmonary. Patients did not receive previously antibiotic prophylaxis, steroids, nor parenteral nutrition. A non-probabilistic sampling was performed.

\section{Variables}

Sociodemographic characteristics such as age, sex, type of bacteria isolated in the infection were evaluated. In addition, NLR and MPV were assessed at the time of clinical suspicion of bacterial infection and culture collection (72 hours before the positivity of the culture) and when the culture was positive.

The microbiology laboratory determined infection by bacterial isolation in blood, urine, or trachea cultures.

\section{Statistical analysis}

Analyzes were carried out with RStudio and IBM ${ }^{\circledR}$ SPSS version 25. Descriptive statistics were used, using tables and graphs representing the absolute and relative values of the qualitative variables and measures of central tendency and dispersion for the quantitative variables. 
The assumption of normality of age, NLR and MPV was verified. The Wilcoxon test was used to compare the mean NLR and MPV between the evaluation moments, while the Mann-Whitney

Table 1 - Clinical characteristics of the patients.

\begin{tabular}{|l|c|}
\hline \multicolumn{1}{|c|}{ Characteristics } & Values \\
\hline Age, mean (SD) & $50(11.66)$ \\
\hline Gender, $\mathrm{n}(\%)$ & $58(78.38)$ \\
Male & $16(21.62)$ \\
Female & $122(100)$ \\
\hline Isolated bacteria, $\mathrm{n}(\%)$ & $18(14.75)$ \\
Pseudomonas aeruginosa & $12(9.84)$ \\
Staphylococcus aureus & $12(9.84)$ \\
Staphylococcus epidermidis & $10(8.2)$ \\
Klebsiella pneumoniae & $9(7.38)$ \\
Klebsiella pneumoniae ESBL & $8(6.56)$ \\
Escherichia coli & $8(6.56)$ \\
Pseudomona aeruginosa KPC & $8(6.56)$ \\
Staphylococcus aureus MR & $8(6.56)$ \\
Staphylococcus hominis & $4(3.28)$ \\
Proteus mirabilis & $3(2.46)$ \\
Enterobacter spp & $3(2.46)$ \\
Enterococcus faecalis & $2(1.64)$ \\
Klebsiella pneumoniae KPC & $2(1.64)$ \\
Staphylococcus warneri & $2(1.64)$ \\
Streptococcus constellatus & $13(10.66)$ \\
Other &
\end{tabular}

$\mathrm{SD}=$ Standard deviation. test was used to compare NLR and MPV by sex and age. Statistical significance was established with a $\mathrm{p}$-value $<0.05$.

\section{RESULTS}

Seventy-four patients were analyzed, with an average age of 50 years; the majority were male, $78,38 \%$.

One hundred twenty-two bacteria were isolated, the most frequent was Pseudomonas aeruginosa $14.75 \%$, followed by Staphylococcus aureus $9.84 \%$ and the same percentage for Staphylococcus epidermidis, Klebsiella pneumoniae $8.20 \%$, Klebsiella pneumoniae ES $\beta$ L 7.38\%, Escherichia coli $6.56 \%$ and the same percentage for Pseudomonas aeruginosa KPC, Staphylococcus aureus MRSA and Staphylococcus hominis. Thus, these bacteria represented $76.25 \%$ of the isolated bacteria (Table 1).

The neutrophil-to-lymphocyte ratio (NLR) was compared three days before the culture and the day of taking the culture, observing significant differences with a p-value of 0.020 . The mean NLR was 18.83 three days before the culture vs 28,62 for the day of cultivation. Therefore, the NLR increased in absolute values by $9.79,51.99 \%$ (Figure 1).

On the other hand, MPV three days before the culture and the day of the culture were compared,
Figure 1 - Comparison of Neutrophil-to-lymphocyte ratio (NLR) by evaluation time.

Note: Based on the Wilcoxon test.

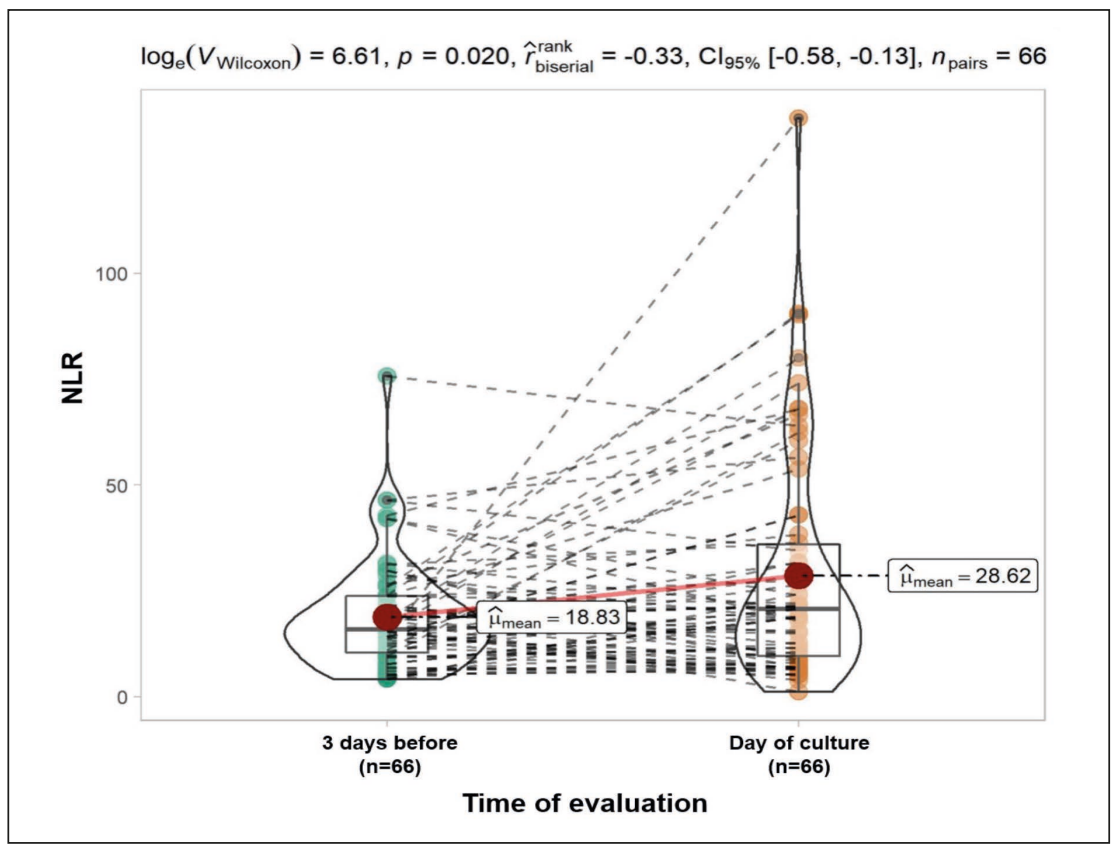




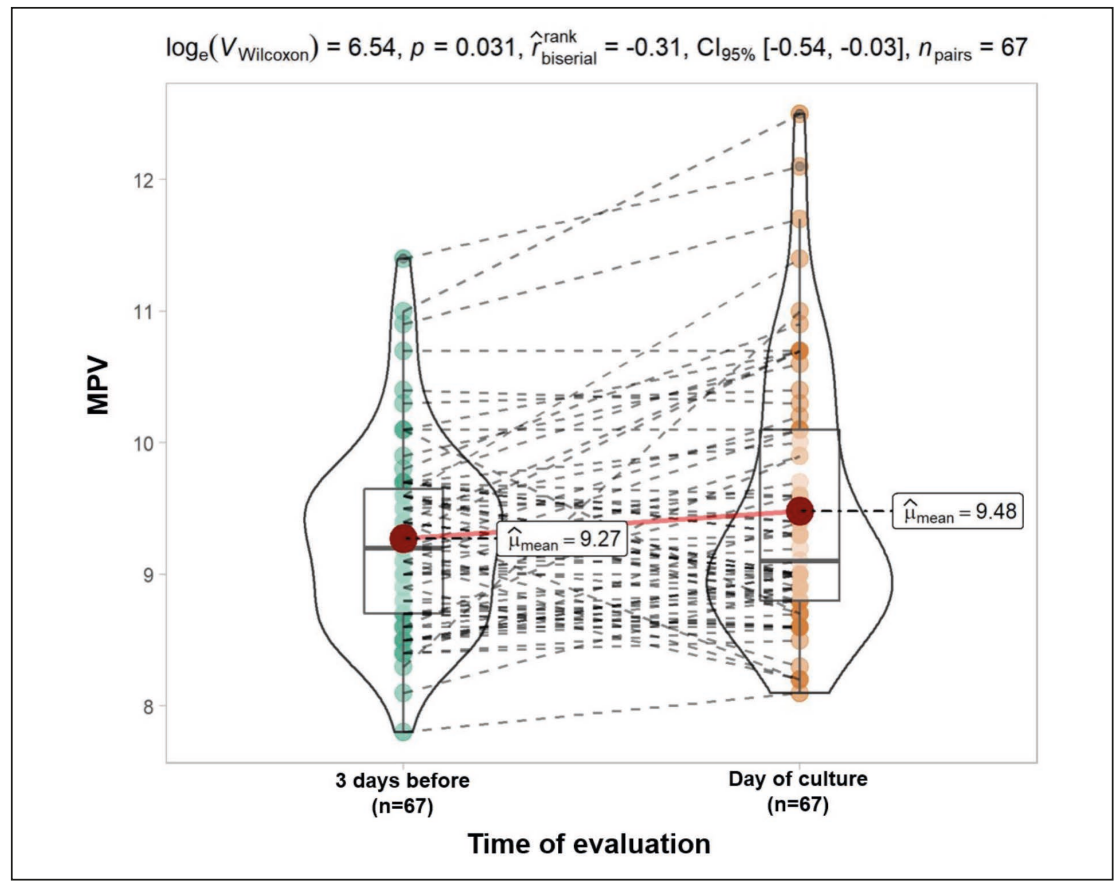

Figure 2 - Comparison of MPV by evaluation time.

Note: Based on the Wilcoxon test.

observing significant differences with p-value 0.031, where the mean MPV was $9.27 \mathrm{fL}$ three days before the culture vs $9.48 \mathrm{fL}$ for culture day (Figure 2).

NLR and MPV were compared at the different evaluation times according to sex and age group; observing for the age group significant differences for the NLR three days before the culture with $\mathrm{p}$ value 0.004 , where the means of NLR of 13.99 for $\leq 50$ years vs 23.12 for $>50$ years; likewise, significant differences were observed for MPV three days before the culture with p-value 0.015, where the means were $9.08 \mathrm{fL}$ for $\leq 50$ years vs $9.45 \mathrm{fL}$ for $>50$ years (Table 2). Other factors were not significant.
When correlating age with NLR and MPV, significance was observed for the different evaluation moments, where the correlation coefficients were 0.403 for age and NLR three days before culture (NLR 3BC), 0.266 for age, and NLR on the day of culture (NLR DC), 0.266 for age and MPV three days before culture (MPV 3BC), and 0.272 for age and MPV on the day of culture (MPV DC); the coefficients were positive. Therefore, there is a direct linear relationship between NLR and MPV with age at the different moments evaluated (Figure 3).

The NLR of the day of the culture was compared by Gram positive and negative, not observing

Table 2 - NLR and MPV comparison according to demographic characteristics.

\begin{tabular}{|c|c|c|c|c|c|c|c|c|}
\hline \multirow{3}{*}{$\begin{array}{l}\text { Demographic } \\
\text { characteristics }\end{array}$} & \multicolumn{4}{|c|}{$N L R$} & \multicolumn{4}{|c|}{$M P V$} \\
\hline & 3 days before & & Cultivation day & & 3 days before & & Cultivation day & \\
\hline & Mean (SD) & p-value & Mean (SD) & p-value & Mean (SD) & p-value & Mean (SD) & $p$-value \\
\hline $\begin{array}{l}\text { Gender } \\
\text { Male } \\
\text { Female }\end{array}$ & $\begin{array}{c}20.35(13.57) \\
13.16(5.55)\end{array}$ & 0.082 & $\begin{array}{l}30.99(28.5) \\
17.11(7.37)\end{array}$ & 0.253 & $\begin{array}{l}9.28(0.74) \\
9.24(0.59)\end{array}$ & 0.682 & $\begin{array}{l}9.69(1.12) \\
9.21(0.76)\end{array}$ & 0.163 \\
\hline $\begin{array}{l}\text { Age group } \\
\leq 50 \text { years } \\
>50 \text { years }\end{array}$ & $\begin{array}{c}13.99(8.25) \\
23.12(14.29)\end{array}$ & $0.004^{*}$ & $\begin{array}{l}23.88(27.66) \\
31.68(24.28)\end{array}$ & 0.062 & $\begin{array}{l}9.08(0.59) \\
9.45(0.77)\end{array}$ & $0.015^{*}$ & $\begin{array}{l}9.44(1.06) \\
9.71(1.06)\end{array}$ & 0.197 \\
\hline
\end{tabular}

$\mathrm{SD}=$ Standard deviation, * significant differences in means, based on the Mann-Whitney test. 
significant differences, however the means of the NLR were 30.11 for Gram positive and 24.89 for Gram negative (Figure 4). The MPV of the day of the culture was also compared by Gram positive and negative, not observing significant differences, however the means of the MPV were $9.73 \mathrm{fL}$ for Gram positive and $9.37 \mathrm{fL}$ for Gram negative (Figure 5).
Figure 3 - Correlation between age, NLR and MPV.

Note: Pearson correlation significance $\mathrm{p}$-value $<{ }^{* * *} 0.001^{* *} 0.01$ ${ }^{*} 0.05$. BC, before culture; $A C$, after culture.
Figure 4 - Comparison of the NLR by the culture day by Gram result.
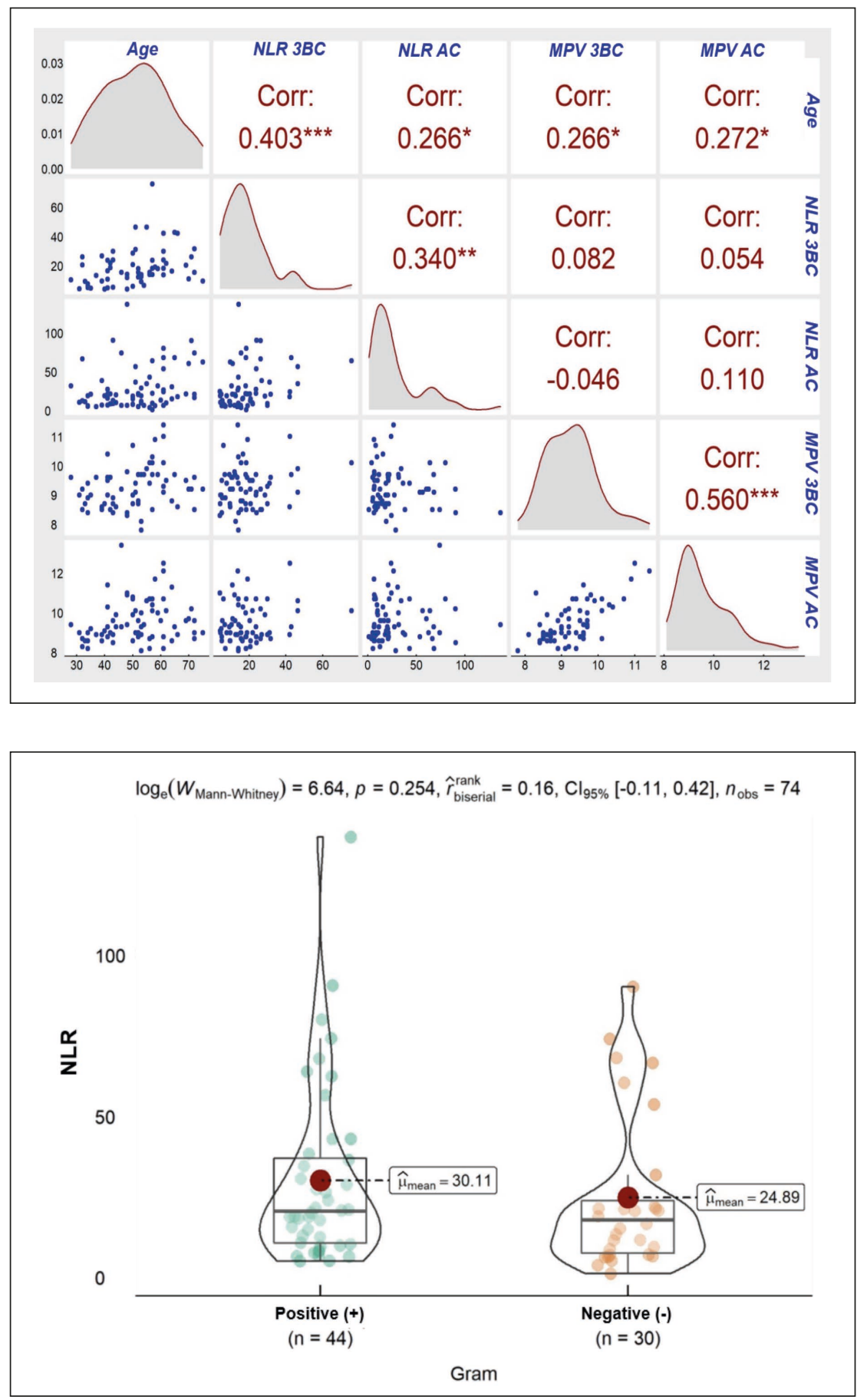


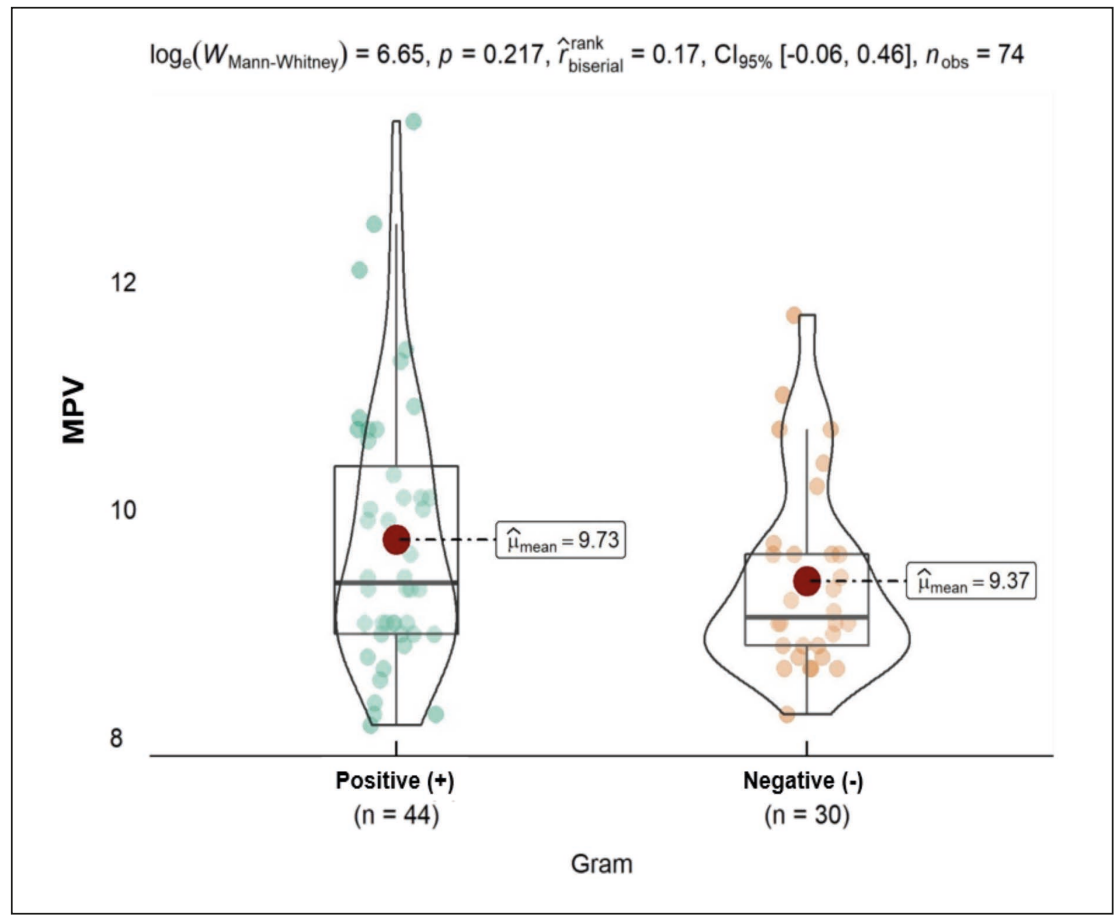

Figure 5 - Comparison of the MPV by the culture day by Gram result.

\section{DISCUSSION}

Our study evaluated the clinical and epidemiological characteristics of patients with COVID-19 and infections associated with in-hospital bacteria and correlated with the neutrophil-to-lymphocyte ratio (NLR) and mean platelet volume (MPV) in patients hospitalized in ICU with COVID-19. In our study, significant differences were found in NLR and MPV between the three days before culture and the day of culture.

During systemic bacterial infections, pathogenassociated molecular patterns (PAMPs) such as lipopolysaccharide (LPS) bind to pattern recognition receptors (PRRs) and activate a response with the production of pro-inflammatory interleukins (TNF alpha, IL6, IL-1, and IL-8), which induces the production and activation of neutrophils. Thus, the decrease in lymphocytes in the blood is due to the marked acceleration of their apoptosis. In addition, cortisol, cytokines, and endotoxins activate and promote the recirculation of CD4, CD8, and other subpopulations from the peripheral blood to affected organs. For this reason, the lymphocytic neutrophilic index has been identified as a prognostic factor for patients with postopera- tive pneumonia since it manages to predict better mortality than other indices such as CURB-65 and C-reactive protein (CRP) in elderly patients with pneumonia [11].

Platelets contain RRPs such as the toll-like receptor (TLR) and the NOD-like receptor (NLR3) that are part of the immune system recognizing molecular patterns of a broad spectrum of infectious agents. In addition, they have antimicrobial activity by storing and releasing alpha granules such as PF4. During infection, pro-inflammatory cytokines, such as IL-6, induce the production of immature thrombocytes that are larger. Therefore, they have a greater volume of granular content and a greater surface area approached by receptors.

Several studies have demonstrated the diagnostic utility of MPV in bacterial infections, among them the one by Vardon-Bounes et al. [12], which studies the kinetics of MPV and determines significance in survival at 90 days of MPV with HR 3.79 and the meta-analysis carried out by Tajarernmuang et al. [13], in 11 studies $(n=3724)$, which aimed to determine if there is an association between MPV and mortality in critically ill patients, finding a significant association with the values 
of day 3. Regarding the prognostic utility in COVID-19, Sertbas et al. [14] conducted a multicenter study with almost 10,000 patients, which shows that with an MPV value> 10.05, it adequately predicts mortality in patients with COVID-19 (OR 5.15, 95\% CI: 4.374-6.067). In the literature review, no reports have been found on the diagnostic value for bacterial infections in patients with $\mathrm{CO}$ VID-19, so this would be the first report.

Regarding NLR, several reports have determined its diagnostic value for bacterial infections. Among them, Ahn et al. [15] demonstrated that NLR has a higher diagnostic value than CRP and neutrophils for pneumonia in 523 patients with heart failure; this reinforced by El-Emshaty et al. [16], who determined that NLR is a marker that can differentiate, even with greater precision than CRP, atypical bacterial pneumonia in 187 patients. The NLR diagnostic value for bacterial infections in COVID-19 patients has not been reported. However, although patients with COVID-19 have a normal white blood cell count, an increase in neutrophils and a decrease in the lymphocyte count would be significantly associated with a bacterial infection in COVID-19.

It is worth mentioning that NLR may have higher levels in some non-infectious diseases. It is reported as a possible prognostic biomarker in acute coronary syndrome and cancer, while the elevation of MPV would be associated with chronic diseases such as diabetes and hypertension [17].

The diagnosis of bacterial co-infection in patients with COVID-19 becomes a challenge. The traditional and current diagnostic criteria of sepsis lose precision since the cytokine storm and frequent thromboembolic episodes in COVID-19 are perfectly simulate a systemic inflammatory response and multi-organ failure [18]. Furthermore, the polypharmacy that these patients receive, such as corticosteroids and tocilizumab, skews the clinical signs that warn about new infections. Therefore, the relevance of finding biomarkers that increase diagnostic certainty and help in the clinical decision to start antibiotic therapy in these patients [19]. Because the NLR and MPV are routinely reported in the routine blood count, they are low cost, easily accessible, and have been obtained before the result of the culture; they are of importance during the pandemic and even more so in developing countries where higher rates of ventilator-associated pneumonia are reported [20].
In a meta-analysis with 3,834 patients, the prevalence of bacterial coinfection in hospitalization and intensive care was evaluated, reporting percentages of 7 and $14 \%$, respectively [21]. Thus, patients with COVID-19 and with suspected bacterial coinfection tend to have a worse prognosis than those without infection, so it is essential to recognize it in a prudent time for a practical approach since inappropriate and indiscriminate treatment in COVID-19 patients can cause Clostridium difficile infection and antimicrobial resistance [22-25]. Our study has severe limitations. First, the sample size is small, making it difficult to extrapolate the results to a significant population. Second, being a small sample, an analysis with a ROC curve was performed to determine the sensitivity of NLR and MPV, but no significant results were obtained. Therefore, the analysis was not included in the study. It is advisable to continue to enrol more patients in the study so that in the future, we can add results on the diagnostic accuracy of the NLR and MPV in the timely diagnosis of bacterial infection in patients with COVID-19.

In conclusion, our study finds significant differences in NLR and MPV between the three days before culture and the day of culture, which may have multiple implications in the diagnostic decision in an early stage of COVID-19 patients evolution. It is advisable to continue to enrol more patients in the study so that in the future, we can add results on the diagnostic accuracy of the NLR and MPV in the timely diagnosis of bacterial infection in patients with COVID-19, as this may be considered a preliminary analysis from a country in Latin America, a region severely affected by COVID-19. As reported in other studies, NLR and MPV were very good independent predictors of lethal outcome [26]. Those studies have demonstrated that the nature of bacteremia influences the mean platelet volume-to-platelet count (MPV / PC) ratio, monocyte-to-lymphocyte ratio (MLR), and platelet-to-lymphocyte ratio (PLR) [26].

\section{Ethical approval}

This is a retrospective study. The hospital board authorized and endorsed this study.

\section{Declaration of competing interest}

None for all authors.

\section{Funding}

There was no specific funding for this project. 


\section{REFERENCES}

[1] Rodriguez-Morales AJ, Cardona-Ospina JA, Gutierrez-Ocampo E, et al. Clinical,laboratory and imaging features of Covid-19: A Systematic Review and MetaAnalysis. Travel Med Infect Dis. 2020; 34, 101623.

[2] Dhama K, Khan S, Tiwari R, et al. Coronavirus Disease 2019-Covid-19. Clin Microbiol Rev. 2020; 33 (4).

[3] Smith L, Karaba SM, Amoah J, et al. Hospital-acquired infections among adult patients admitted for coronavirus disease 2019 (Covid-19). Infect Control Hosp Epidemiol. 2021, 1-4.

[4] Yao C, Bora SA, Parimon T, et al. Cell-Type-Specific Immune Dysregulation in Severely Ill Covid-19 Patients. Cell Reports. 2021; 34 (1), 108590.

[5] Rutkowska E, Kwiecień I, Kulik K, et al. Usefulness of the new hematological parameter: reactive lymphocytes re-lymp with flow cytometry markers of inflammation in Covid-19. Cells. 2021; 10 (1), 82.

[6] Lagunas-Rangel FA. Neutrophil-to-lymphocyte ratio and lymphocyte-to-c-reactive protein ratio in patients with severe coronavirus disease 2019 (Covid-19): A Meta-Analysis. J Med Virol. 2020; 92 (10), 1733-4.

[7] Usul E, Şan 1, Bekgöz B, and Şahin A. Role of hematological parameters in Covid-19 Patients in the emergency room. Biomark Med. 2020; 14 (13), 1207-15.

[8] Zhang Y, Wu L, Yang J, Zhou C, and Liu Y. A nomoGram-based prediction for severe pneumonia in patients with Coronavirus Disease 2019 (Covid-19). Infect Drug Resist. 2020; 13, 3575-3582.

[9] Liu Y, Du X, Chen J, et al. Neutrophil-to-Lymphocyte ratio as an independent risk factor for mortality in hospitalized patients with Covid-19. J Infect. 2020; 81 (1), e6-e12.

[10] Belice T, Demir I, and Yüksel A. Role of neutrophillymphocyte-ratio in the mortality of males diagnosed with Covid-19. Iran J Microbiol. 2020; 12 (3), 194-7.

[11] Cataudella E, Giraffa CM, Di Marca S, et al. Neutrophil-to-Lymphocyte Ratio: an emerging marker predicting prognosis in elderly adults with community-acquired pneumonia. J Am Geriatr Soc. 2017; 65 (8), 1796-801.

[12] Vardon-Bounes F, Gratacap MP, Groyer S, et al. Kinetics of mean platelet volume predicts mortality in patients with septic shock. PLoS One. 2019; 14 (10), e0223553.

[13] Tajarernmuang P, Phrommintikul A, Limsukon A, Pothirat $\mathrm{C}$, and Chittawatanarat $\mathrm{K}$. The role of mean platelet volume as a predictor of mortality in critically ill patients: a systematic review and meta-analysis. Crit Care Res Pract. 2016; 2016, 4370834.

[14] Sertbaş M, Dağc1 S, Kızılay V, et al. Mean Platelet Volume as an Early Predictor for the Complication of
Coronavirus Disease 19. Haydarpasa Numune Med J. 2021; 61 (2), 177-82.

[15] Ahn JM, Hwang SO, Moon JS, Lee SJ, and Cha YS. Predictive value of the neutrophil-to-lymphocyte ratio for the diagnosis of pneumonia in normothermic dyspneic patients with chronic heart failure in the emergency department. J Emerg Med. 2020; 58 (6), 892-901.

[16] El-Emshaty W, Mashaly M, Moawad A, Elgamal M, and Hewidy A. Diagnostic value of neutrophil-lymphocyte ratio versus c-reactive protein in discrimination between different pathogens causing community-acquired pneumonia. Compar Clin Pathol. 2017; 26 (4), 757-65.

[17] Kurtul A, Yarlioglues M, Murat SN, et al. Predictors of chronic total occlusion in nonculprit artery in patients with acute coronary syndrome: mean platelet volume and uric acid. Angiology. 2015; 66 (6), 553-9.

[18] Kumar V. Toll-Like Receptors in sepsis-associated cytokine storm and their endogenous negative regulators as future immunomodulatory targets. Intern Immunopharmacol. 2020; 89 (Pt B), 107087.

[19] Schuetz P, Amin DN, and Greenwald JL. Role of Procalcitonin in managing adult patients with respiratory tract infections. Chest. 2012; 141 (4), 1063-73.

[20] Kumar Gothwal S, Singh VB, Shrivastava M, et al. Complete blood-count-based inflammatory score (cbcs) of covid-19 patients at tertiary care center. Alter Ther Health Med. 2021; 27 (S1), 18-24.

[21] Lansbury L, Lim B, Baskaran V, and Lim WS. CoInfections in People with Covid-19: A Systematic Review and Meta-Analysis. J Infect. 2020; 81 (2), 266-75.

[22] Piacenti FJ, and Leuthner KD. Antimicrobial stewardship and Clostridium difficile-associated diarrhea. J Pharm Pract. 2013; 26 (5), 506-13.

[23] Álvarez-Moreno C, Valderrama-Beltrán S, and Rodriguez-Morales AJ. Implications of antibiotic use during the covid-19 pandemic: the example of associated antimicrobial resistance in Latin America. Antibiotics (Basel, Switzerland) 2021; 10 (3).

[24] Khor WP, Olaoye O, D'Arcy N, et al. The need for ongoing antimicrobial stewardship during the covid-19 pandemic and actionable recommendations. Antibiotics (Basel). 2020; 9 (12).

[25] Lai CC, Wang CY, and Hsueh PR. Co-infections among patients with covid-19: the need for combination therapy with non-anti-sars-cov-2 agents? J Microbiol Immunol Infect. 2020; 53 (4), 505-12.

[26] Djordjevic D, Rondovic G, Surbatovic M, et al. Neutrophil-to-lymphocyte ratio, monocyte-to-lymphocyte ratio, platelet-to-lymphocyte ratio, and mean platelet volume-to-platelet count ratio as biomarkers in critically ill and injured patients: which ratio to choose to predict outcome and nature of bacteremia? Med Inflam. 2018; 2018, 3758068. 